\title{
Situación de enteroparasitosis en niños menores de 5 años en la Comunidad de Villa Trinidad
}

\author{
Situation of enteroparasitosis in children under 5 years in the \\ Community of Villa Trinidad

\section{Situação da enteroparasitose em crianças menores de 5 anos na Comunidade de Villa Trinidad}

\author{
Andree Joseline Michel Martinez \\ a.n.d.r.e.e@hotmail.com
}

Recibido enero 2019 / Revisión febrero 2019 / Aceptado 1 de mayo 2019

\begin{abstract}
RESUMEN
Introducción: La parasitosis intestinal es un problema común y de preocupación en los países subdesarrollados, en Villa Trinidad debido a las bajas condiciones de vida, por falta de saneamiento básico, inadecuada higiene de manos y consumo de alimentos contaminados. Objetivo: Determinar la situación actual de enteroparasitosis en niños menores de 5 años en la Comunidad de Villa Trinidad. Materiales y Método: El tipo de estudio fue observacional, retrospectivo, descriptivo y de corte transversal, cada participante brindo muestra de heces para análisis coproparasitológico previa consulta, las muestras fecales fueron estudiadas mediante examen directo en solución salina fisiológica y técnica modificada Ritchie en laboratorios de INLASA. Resultados: Se evaluaron 19 niños entre 0-5 años de edad, de ellos: 9 masculinos y 9 femeninos, 15 de estos presentaron parásitos en el examen coproparasitològico. El hallazgo de parasitosis intestinales general fue Giardia intestinalis, Complejo Entamoeba histolytica y dispar, Áscaris lumbricoides, Entamoeba coli, Lodomoeba butschlii, Endolimax nana y Blastocystis hominis. Se diagnosticaron parasitosis de 7 especies diferentes, de ellos 9 eran protozoarios y 1 helminto. Conclusión: Se determinó una alta prevalencia de parasitosis intestinal en niños menores de 5 años, el poliparasitismo fue superior y ligado a medidas de saneamiento ambiental a diferencia del uniparasitismo que se encontró más relacionado al consumo de agua y otros.
\end{abstract}

Palabras clave: Parásitos; prevalencia; saneamiento; higiene

Introduction: Intestinal parasitosis is a common problem and of concern in underdeveloped countries, in Villa Trinidad due to low living conditions, lack of basic sanitation, inadequate hand hygiene and consumption of contaminated food. Objective: To determine the current situation of enteroparasitosis in children under 5 years of age in the Villa Trinidad Community. Materials and Method: The type of study was observational, retrospective, descriptive and cross-sectional, each participant provided stool sample for coproparasitological analysis after consultation, and fecal samples were studied by direct examination in physiological saline and modified Ritchie technique in laboratories of INLASA. Results: 19 children between 0-5 years of age were evaluated, of them: 9 male and 9 female, 15 of these presented parasites in the coproparasitological examination. The finding of general intestinal parasitosis was Giardia intestinalis, Entamoeba histolytic a and dispar complex, Ascaris lumbricoides, Entamoeba coli, Lodomoeba butschlii, Endolimax nana and Blastocystis hominis. Parasitic diseases of 7 different species were diagnosed, 9 of them were protozoan and 1 helminth. Conclusion: A high prevalence of intestinal parasitosis was determined in children younger than 5 years, polyparasitism was higher and linked to environmental sanitation measures, unlike uniparasitism, which was found to be more related to water consumption and others.
\end{abstract}

Key words: Parasites; prevalence; sanitation; hygiene 
AM Universidad Mayor de San Andrés Bolivia

\section{RESUMO}

Introdução: A parasitose intestinal é um problema comum e preocupante em países subdesenvolvidos, em Villa Trinidad, devido às baixas condições de vida, falta de saneamento básico, higiene inadequada das mãos e consumo de alimentos contaminados. Objetivo: Determinar a situação atual da enteroparasitose em crianças menores de 5 anos na Comunidade Villa Trinidad. Materiais e Métodos: $O$ tipo de estudo foi observacional, retrospectivo, descritivo e transversal; cada participante forneceu amostra de fezes para análise coproparasitológica após consulta; as amostras fecais foram estudadas por exame direto em soro fisiológico e técnica de Ritchie modificada em laboratórios de INLASA. Resultados: foram avaliadas 19 crianças entre 0-5 anos de idade, sendo 9 do sexo masculino e 9 do feminino, 15 delas apresentaram parasitas no exame coproparasitológico. Os achados de parasitose intestinal geral foram Giardia intestinalis, Entamoeba histolytica e dispar, Ascaris lumbricoides, Entamoeba coli, Lodomoeba butschlii, Endolimax nana e Blastocystis hominis. Foram diagnosticadas doenças parasitárias de 7 espécies diferentes, 9 delas protozoários e 1 helminto. Conclusão: Foi determinada alta prevalência de parasitose intestinal em crianças menores de 5 anos; o polparasitismo foi maior e vinculado a medidas de saneamento ambiental, diferentemente do uniparasitismo, que se mostrou mais relacionado ao consumo de água e outros.

Palavras-chave: Parasites; prevalência; saneamento higiene

\section{INTRODUCCIÓN}

as enfermedades parasitarias están

$\mathrm{L}$ ampliamente distribuidas por el mundo, causan un gran impacto ya que inciden de manera importante sobre la salud, la esperanza de vida y la productividad de millones de personas. Las infecciones parasitarias son un problema de salud pública, debido a que suelen causar anemia por deficiencia de hierro, mala absorción de nutrientes y diarrea, entre las principales afecciones. Tradicionalmente las parasitosis intestinales se han relacionado con países en vías de desarrollo con poblaciones de escasos recursos económicos y deficientes condiciones higiénico-sanitarias. Frecuentemente, la elevada prevalencia de parasitosis está relacionada con la contaminación fecal del agua de consumo humano y del suelo o de los alimentos unida a deficientes condiciones sanitarias y socioculturales.

Los factores climáticos y socioeconómicos determinan en gran manera la distribución geográfica de las enfermedades parasitarias que afectan a personas de todas las edades, pero especialmente a los niños pudiendo causar desde molestias leves hasta la muerte (1).

Según la Organización Mundial de la Salud, la prevalencia de las enteroparasitosis en América Latina oscila entre 20 y $30 \%$ de la población general y 60 a $80 \%$ para poblaciones con alta endemia. En niños menores de cinco años de edad, las cifras oficiales de mortalidad refieren que la mayor parte de las defunciones fueron causadas por enfermedades infecciosas incluyendo las parasitarias, que representaron $13 \%$ del total. Entre las enfermedades infecciosas las intestinales constituyeron el grupo más importante (7\%).

Las enfermedades infecciosas intestinales son aquellas enfermedades producidas por endoparásitos y son las que mayor impacto tienen sobre la salud del huésped. Entre los endoparásitos más comunes que afectan al ser humano tenemos G. lamblia, Criptosporidium, Blastocystis hominis, Ascaris lumbricoides $\mathrm{y}$ Enterobuis vermicularis, entre otros $(1,2)$. 
Los resultados de los trabajos recopilados desde el año 2006 muestran que la prevalencia de nematodos intestinales es mayor en zonas geográficas con ata temperatura y humedad, aspecto clásico del país y esto es reconocido mundialmente (3). e. En Perú, se han realizado estudios de parasitosis intestinal, desde el punto de vista de prevalencia, incidencia, factores de riesgo, entre otros.

En el 2013, se realizó un estudio acerca de la prevalencia e intensidad de infección de parasitosis intestinal en niños de 4 años de 45 jardines de infancia de San Juan de Lurigancho, Lima - Perú. Se encontró biparasitismo y triparasitismo, siendo Giardia lamblia el protozoo más frecuente $(37,54 \%)$, Hymenolepis nana $(21,59 \%)$. Concluyen que la prevalencia varía de acuerdo a las zonas y sus características socioeconómicas. (4).

El año 1985 el Ministerio de Prevención Social y Salud Publica decidió aplicar un programa de control de las parasitosis en niños de 2 a 12 años con cobertura contra los helmintos más importantes (ascaris lumbricoides, trichuris trichura, anquilostomideos) al administrar Mebendazol en dosis única de $200 \mathrm{mg}$ en zonas con clima frio, $300 \mathrm{mg}$ con clima templado y $400 \mathrm{mg}$ con clima cálido; ello se extendió por cinco años entre 1986 y 1990; según los registros oficiales luego de ese esquema disminuyó la prevalencia de helmintiasis en particular en zonas urbanizadas, pero en menor grado en áreas periurbanas $(5,6)$.

En la zona tropical, los enteroparasitos alcanzan mayor relevancia lo cual se refleja en el aislamiento frecuente de Ascaris lumbricoides con cifras generalmente mayores de 60\% (15 a 96\%) de Trichuris trichiura (5 a 87\%) y de Uncinarias (1 al 84\%). En cuanto a protozoarios, ellos tienen un comportamiento peculiar porque su prevalencia mayor o menor es dependiente de otros factores condicionantes de estas infecciones (higiene personal precaria, carencia de servicios básicos, pobreza, etc.) (7).

Las infecciones por parásitos intestinales son endémicas $\mathrm{y}$ han sido descritas como parte de una de las mayores causas de morbi-mortalidad (8). Estas afectan principalmente a la población infantil debido a su inmadurez inmunológica y poco desarrollo de hábitos higiénicos, particularmente en las áreas rurales, barrios pobres y marginalizados donde habitan personas vulnerables en un ciclo de pobreza.

Existen muchos factores de riesgo que condicionan a padecer de múltiples enfermedades entre las que están las siguientes: insalubridad, consumo de agua contaminada, inadecuada higiene personal y de los alimentos, manos sucias, además se agrega el desconocimiento de los habitantes sobre las diversas causas y consecuencias que puede acarrear la parasitosis intestinal $(3,6)$.

\section{MATERIALES Y MÉTODO}

e realizó un estudio de tipo

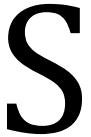
descriptivo. El universo constituyo un total de 19 niños menores de 5 años en esta comunidad. Tomando en cuenta que el tamaño muestral calculado es de 18 niños de un total de 19, al no existir gran diferencia numérica se decidió realizar al total de niños menores de 5 años.

Los criterios de inclusión fueron: niños menores de 5 años de ambos sexos cumplidos hasta el mes de junio de la presente gestión que viven en la Comunidad de Villa Trinidad. Niños cuyos padres o tutores a cargo autoricen a ser partícipes del estudio. Criterios de exclusión: Niños mayores de 5 años de la Comunidad de Villa Trinidad. Niños de 
permanencia temporánea en la Comunidad de Villa Trinidad. Todo aquel que rechace ser parte del estudio. Niños mayores de 5 años que tras detectar esta patología se encuentran con tratamiento antiparasitario en el momento del estudio.

Se realizó la recolección de muestra de materia fecal y aplicación de una encuesta relacionada con el saneamiento ambiental e higiene de los individuos (estructura sanitaria de las viviendas, tipo de abastecimiento de agua, eliminación de basuras y excretas, condiciones de higiene para la preparación y consumo de alimentos y hábitos de higiene personal y colectiva). En cuanto al procesamiento de las muestras se realizó un coproparasitológico directo simple en el
INLASA con posterior entrega de resultados a madres de familia, tratamiento y charla educativa a la comunidad

\section{RESULTADOS Y DISCUSIÓN}

os parásitos más comunes T observados tras el examen coproparasitológico. Dentro de los parásitos patógenos el que más se presento fue Giardia intestinales, seguido de Ascaris lumbricoides con 3 casos y por último el Complejo Entamoeba histolytica y dispar con un caso. Dentro de los parásitos no patógenos el que más se presento fue Entamoeba coli la par de Iodomoeba butschlii. Se observó gran cantidad de infectados por amebas.

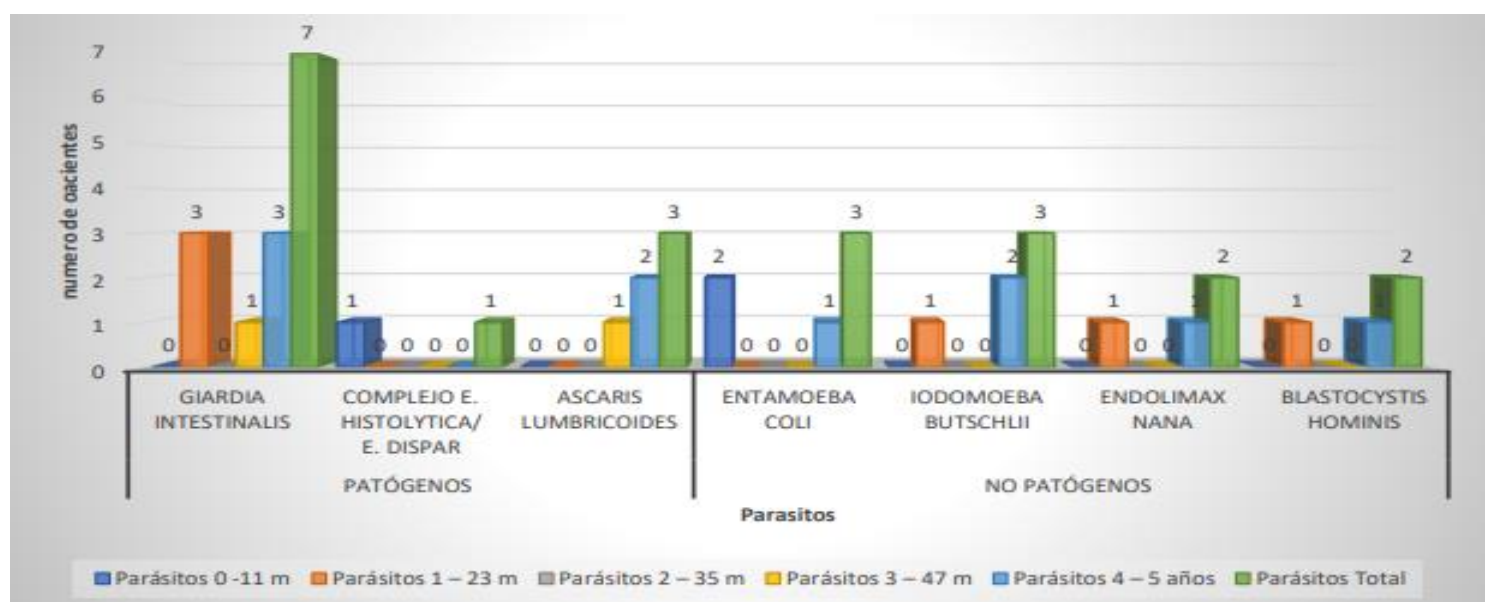

Gráfico 1. Enteroparásitos según edades encontrados en niños menores de 5 años de la comunidad de Villa Trinidad durante el tercer trimestre del 2019.

El mayor número de niños realiza la eliminación de excretas por pozo ciego y este grupo fue el que presentó mayor cantidad de uniparasitosis. 


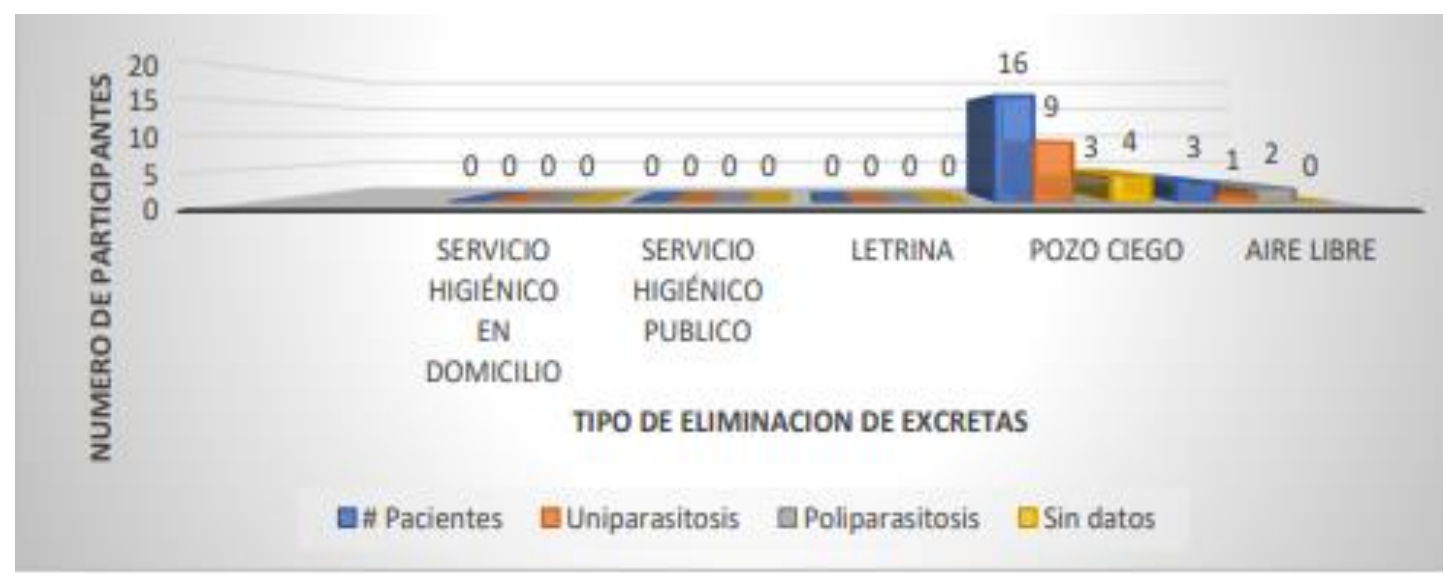

Gráfico 2. Eliminación de excretas con relación a cantidad de enteroparásitos identificados en coproparasitológico de niños menores de 5 años.

La mayor parte de los niños consume agua directamente de la fuente de agua, pero a la par en este grupo existe mayor grupo de niños con uniparasitosis.

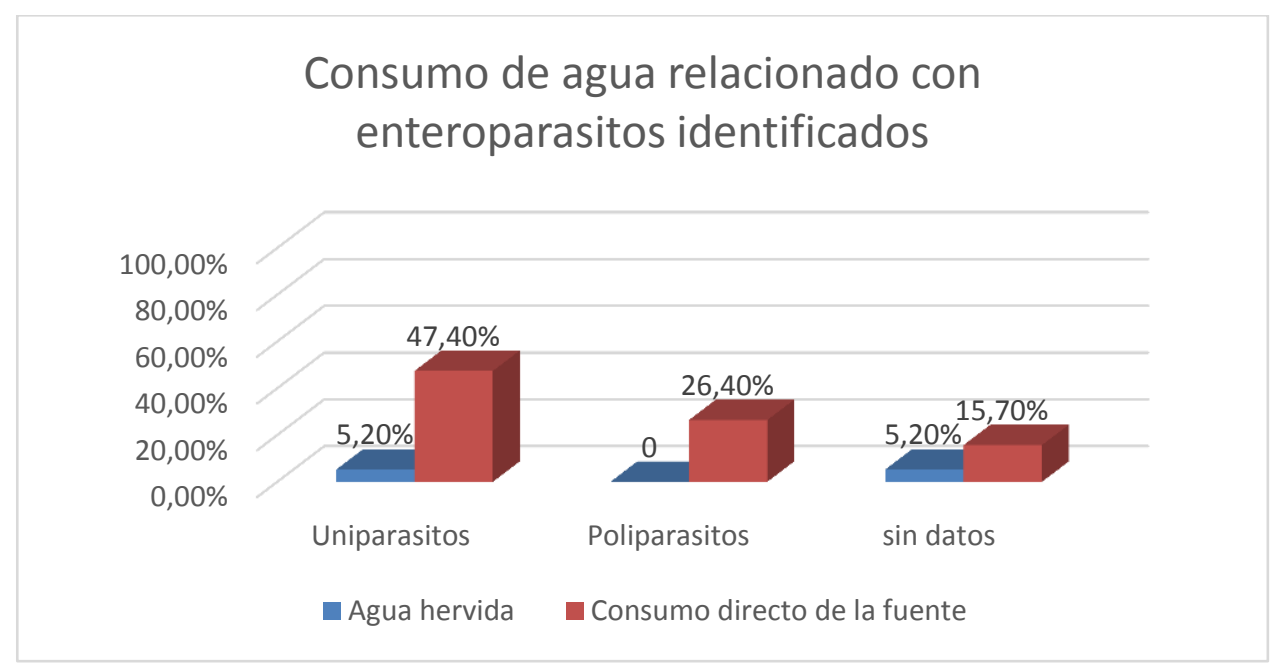

Gráfico 3. Consumo de agua hervida con relación a cantidad de enteroparàsitos identificados en niños menores de 5 años.

\section{Discusión}

Este trabajo realizado en la comunidad de Villa Trinidad dependiente del cantón de Chicaloma, en Bolivia brinda una aproximación a la situación actual del parasitismo intestinal en los niños menores de 5 años de esta localidad y verifica ciertas condiciones predisponentes para la existencia de enfermedades infecciosas intestinales.

Se encontró la presencia de Giardia intestinalis, Complejo Entamoeba histolytica y dispar, Ascaris lumbricoides, Entamoeba coli, Iodomoeba butschlii, Endolimax nana y Blastocystis hominis.

El parasito que se presentó con mayor frecuencia fue Giardia intestinalis que describe que es más común en niños que en adultos posiblemente por el poco desarrollo de hábitos higiénicos adquiridos a estas edades. Los protozoos de mayor frecuencia hallados fueron Giardia lamblia, Entamoeba coli y Iodomoeba butschlii. El alto porcentaje de 
parasitados por protozoarios con respecto a los helmintos puede atribuirse a que las condiciones climáticas de esta comunidad a diferencia de todas las demás es fría misma que no genera un hábitat inapropiado para poder mantener su mecanismo de transmisión de acuerdo con sus ciclos evolutivos $(1,4,6)$. Los resultados mostraron una población con bajos ingresos que en su mayoría tienen limitado acceso a servicios públicos (agua potable, alcantarilla y recolección de basura), con una disponibilidad de agua potable inexistente en esta comunidad, el abastecimiento que se instauro mediante prolongaciones provenientes de aguas subterráneas es la que presento mayor número de poliparasitosis $\mathrm{y}$ las que provenientes del rio se vieron ligadas al uniparasitismo, podríamos atribuir todo esto al desecho de basura al rio, mismo que condiciona la contaminación de agua y la reinfección (9).

La incidencia de enfermedades diarreicas en niños se presenta más frecuente por malos hábitos de higiene de manos para el consumo de alimentos, el consumo de agua directa de la fuente y sin hervir condiciona el desarrollo de enteroparasitosis.

\section{CONCLUSIONES}

$\mathrm{E}$ $n$ concordancia con el objetivo planteado el cual fue determinar la situación actual de enteroparasitosis se obtuvo que el parasitismo intestinal en los círculos infantiles menores a 5 años tiene una elevada prevalencia, ya que aproximadamente más de la mitad de los niños estudiados se encontraban con enteroparásitos en el estudio coproparasitológico.

Estos resultados fueron más frecuentes en los niños de límites de edad del grupo estudiado (de 1 año a 23 meses y de 4 a 5 años), predominando las infecciones por protozoarios $y$ asociaciones de parásitos en varios casos.

El protozoo patógeno más común en este grupo fue Giardia intestinalis tanto en grupos etarios como en general, seguido de los no patógenos como ser Entamoeba coli y Iodomoeba butschlii. El Poliparasitismo se presentó vinculado al consumo de prolongaciones de aguas subterráneas y al desecho de excretas al aire libre y el Uniparasitismo al desecho de excretas mediante pozo ciego. El problema de estas afecciones y su transmisión constituye uno problemas epidemiológicos fundamentales, que podría ser abordado con un enfoque más integral y científico, lo que permitirá reducirlo considerablemente.

- Conflicto de intereses: Ninguno declarado por la autora.

- Financiación: Autofinanciado.

- Agradecimientos: Ninguno declarado por la autora.

\section{REFERENCIAS BIBLIOGRÁFICAS}

1. Botero D. Persistencia de parasitosis intestinales endémicas en América Latina. Bull of Sanit Panam. 1981; 90:39-47

2. Mehraj V, Hatcher J, Akhtar S, Rafique G, Beg $M$ A. Prevalence and factors associated with intestinal parasitic infection among children in an urban slum of Karachi. Plosone 2008; 3 (11):3680 - 3682

3. Mollinedo S, Prieto C. Parasitismo intestinal. 1aㅡ ed. Bolivia: Elite; 2006; 2553

4. Pérez J, Hernández M, Pisos E, Carranza C, Castillo M, Aparicio P. Tratamiento de las enfermedades parasitarias (I): Protozoosis.Inf Ter Sist NAc Salud. 2007; 31:3-16

5. Acuña A, Calegari L, Curto S, Lindner C, Rosa R, Salvatella R. Helmintiasis intestinales: manejo de la geohelmintiasis. Montevideo: MSP, OPS, 2003. 
6. Devera R, Requena I, Tudesco RM, Sandoval M, Velásquez V, Blanco Y. Parasitosis intestinales y condiciones socio-sanitarias en un barrio de Soledad, estado Anzoátegui, Venezuela. SABER. 2010; 22 (1)

7. Olalla R, Tercero J. Parasitosis comunes internas y externas. Ámbito Farmacéutico 2016; 30, (4): 33-38

8. Dawes J, Rowley J. La Salud en las Américas. Organización Panamericana de la Salud. Organización Mundial de la Salud. Washington D.C.; 2002. Publicación Científica y Técnica $\mathrm{N}^{\circ} 587$. Consultado el 12 de febrero de 2018 disponible en: https://www.paho.org/salud-en-lasamericas-2017/wpcontent/uploads/2017/09/PrintVersion-Spanish.pdf
9. Londoño ÁL, Mejía S, Gómez Marín JE. Prevalencia y Factores de Riesgo Asociados a Parasitismo Intestinal en Preescolares de Zona Urbana en Calarcá, Colombia. Rev. Sal Pub (Internet) 2009 (Consultado 20 enero 2018); 11(1):72-81. Disponible https://www.redalyc.org/pdf/422/422 11108.pdf 